\title{
A High Resolution, Single Bunch, Beam Profile Monitor
}

\author{
J. Norem*

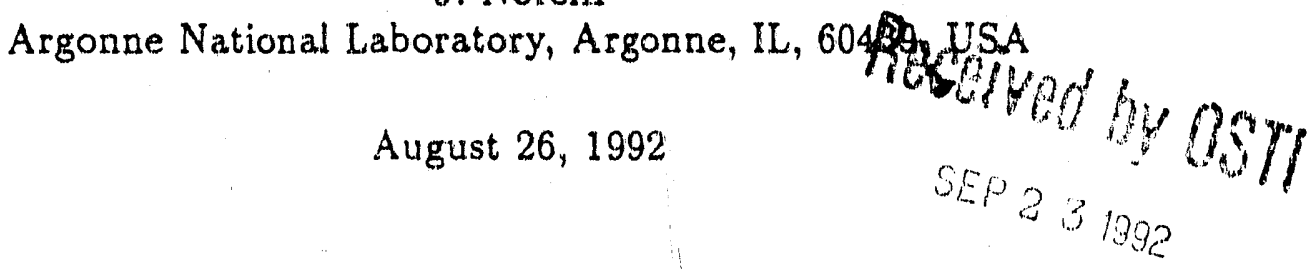

INTROODUCTION

Efficient linear colliders require very small beam spots to produce high luminosities with reasonable input power, which limits the number of electrons which can be accelerated to high energies[1]. The small beams, in turn, require high precision and stability in all accelerator components. Producing, monitoring and maintaining beams of the required quality has been, and will continue to be, difficult. A beam monitoring system which could be used to measure beam profile, size and stability at the final focus of a beamline or collider has been developed and is described here.[2] The system uses nonimagirg bremsstrahlung optics. The immediate use for this system would be examining the final focus spot at the SLAC/FFTB.[3]

The primary alternatives to this technique are those proposed by $P$. Chen / J. Buon, $[4]$ which analyses the energy and angular distributions of ion recoils to determine the aspect ratio of the electron bunch, and a method proposed by Shintake, [5] which measures intensity variation of compton backscattered photons as the beam is moved across a pattern of standing waves produced by a laser.

\section{METHOD}

The system, Figure 1, consists of a Bremsstrahlung radiator at the focus of the electron beam, a single sided collimator to produce a bremsstrahlung shadow, and a slit and detector system to measure the shape of the shadow edge.[2] The diagnostic slit could be either tilted, as shown, or parallel with the primary collimator. The sharpness of the shadow is inversely proportional to the size of the spot at the bremsstrahlung source. Sweeping magnets and shielding are required to disperse and absorb electron and photon backgrounds. The linear dimensions are not critical. The bremsstrahlung photons would be detected using a Cerenkov counter preceeded by a pair converter.

The optics of the system can be shown by plotting bearn phase space at the focus, at the collimator and at the detector downstream, shown in Fig. 2. By moving the detector

-Work supported by the U. S. Department of Energy, Division of High Energy Physics, Contract W-31-109-ENG-38. 
slit back and forth it is possible to measure the shadow width, and hence the beam profile. Moving the primary collimator alters the initial $x^{\prime}$ values, thus the whole phase space density distribution, $\rho\left(x, x^{\prime}, y, y^{\prime}, t\right)$, can be measured.

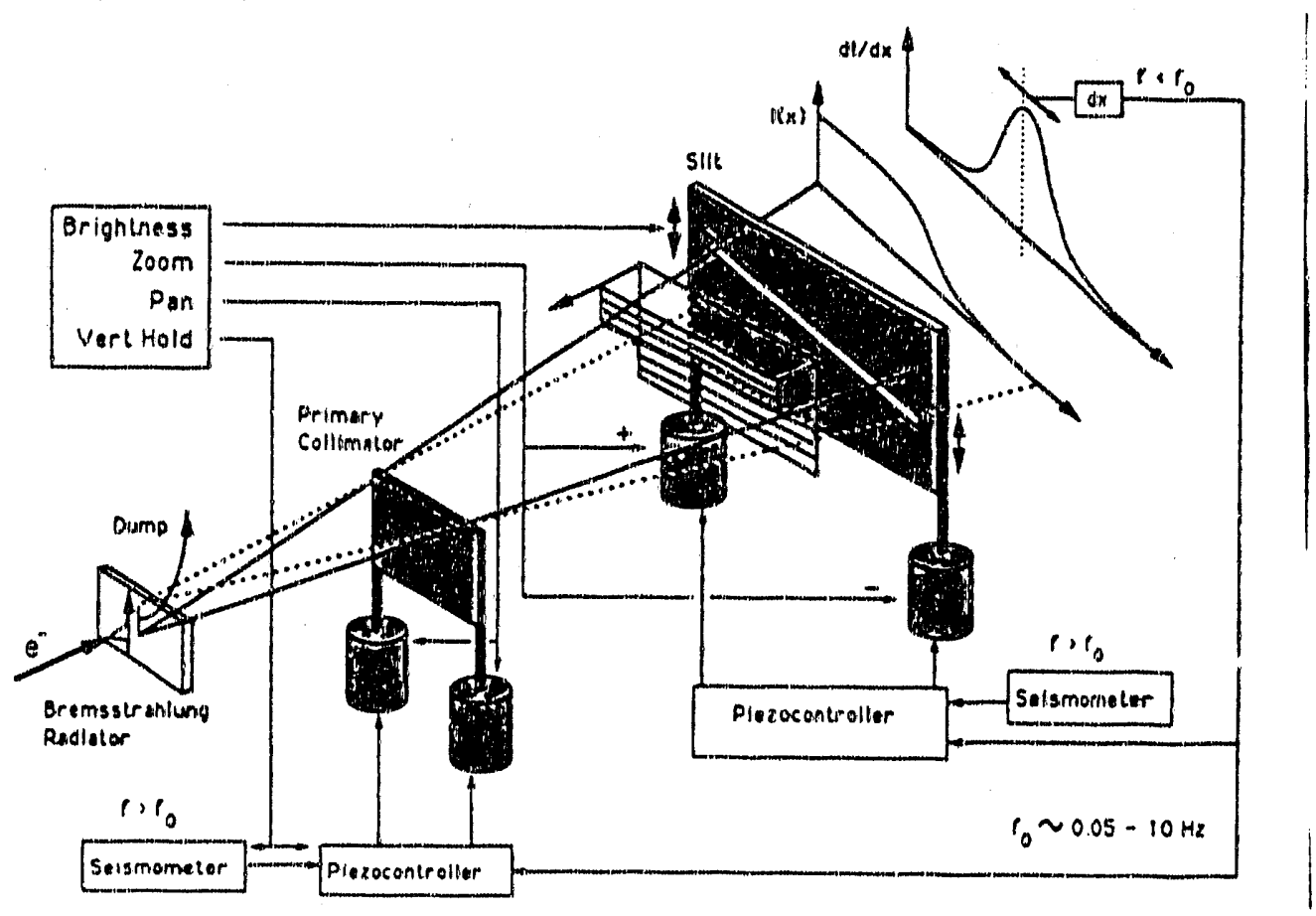

- Figure 1. The system consists of bremsstrahlung radiator, collimator and slit. Detectors and shielding are not shown.

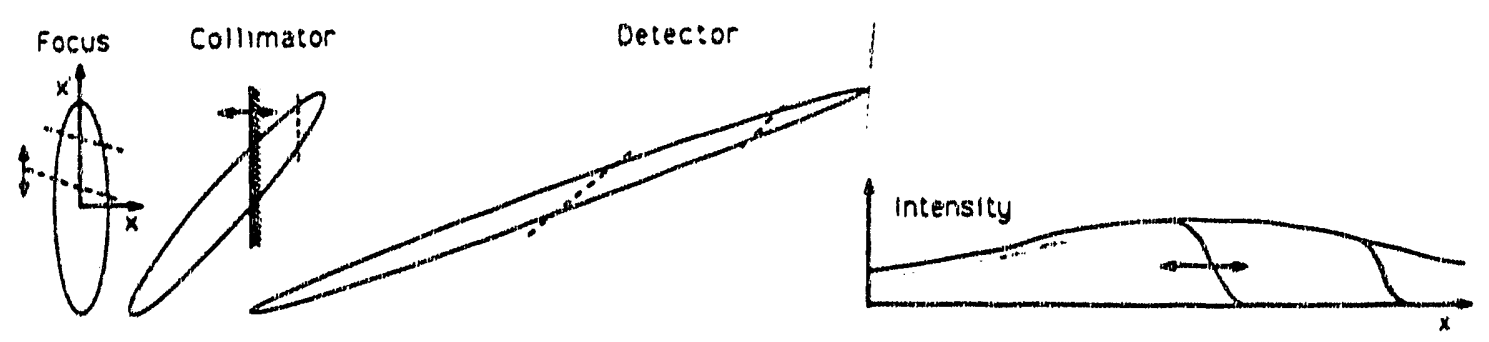

$\square$ Figure 2. Phase space of the systern. not to scale. The collinator produces an edge in the phase space distribution which is measured downstream. A projection in $x$ space shows the observed profile.

\section{LIMITATIONS}

The ultimate resolution of this system is limited by Fresnel diffraction.[6] This limit can be approximated by considering a virtual slit at the primary collinator location, where

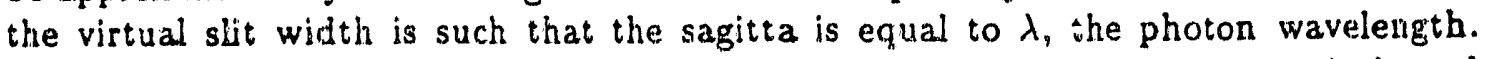
If the source to collimator distance is $a$, the collimator to detector distance is $b$, and $b \gg a$, the expression for the sagitta $\lambda=s^{2} / 2 a$ gives the virtual slit wicth, $s=\sqrt{2 a \lambda}$, Fig 3. The angular diffraction width is then $\sim \lambda / s$ and the limiting resolution is 
$\sim(\lambda / s) a=\sqrt{\lambda a / 2}$, roughly the geometric mean of the beamline dimensions, $(1-10$ $\mathrm{m})$, and the photon wavelength, $\left(\sim 10^{-16} \mathrm{~m}\right)$.
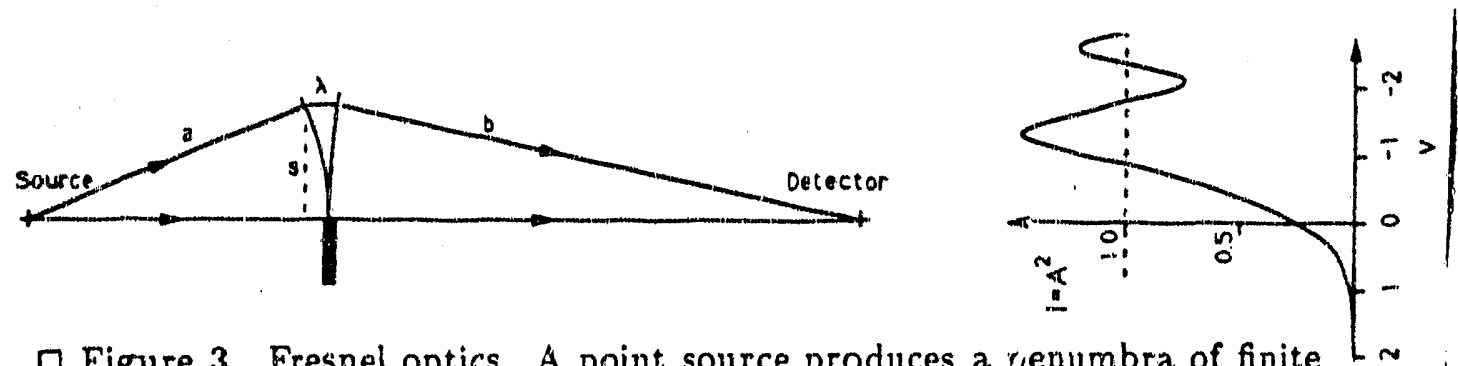

Figure 3. Fresnel optics. A point source produces a yenumbra of finite $L_{\text {r }}$ width.

The precise calculation the diffraction pattern is done for monochromatic light in most optics books. Integrating the intensity from all paths requires solving the Fresnel integrals, the solution of which generates the Cornu spiral. If the intensity of diffraction pattern on a screen is given by $F(y)$, the width of the pattern is a function of

$$
y=v \sqrt{\frac{\lambda a(a+b)}{2 b}},
$$

which gives the dimensions of diffraction patterns on a screen in terms of $v$, the dimensionless variable used to evaluate the Fresnel integrals, and $a=30, b=30 \mathrm{~m}$, and $\lambda .(6]$ The resolution of the systern is determined by incoherently adding the diffraction images produced by the bremsstrahlung spectrum as seen by the detector. The acceptance of the detector has been evaluated using EGS4 and and a more specialized monte carlo program which generates a bremsstrahlung spectrum, computes pair production and subsequently evaluates multiple scattering.[7] The detected FFTB spectrum, which depends somewhat on position and angle cuts, is shown in Fig. 4 for minimum detected electron energy of $15 \mathrm{MeV}$ a.rd maximum angle of $2^{\circ}$. This note defines a resolution function as the derivative of this sum of diffraction images and this is shown in Fig 5 , for the highest resolution possible with the SLAC line. This curve, which is nongaussian, is the effective shape of a beam at the bremsstrahlung radiator in the limit of a zero width slit at the detector. The width of the resolution function depends on energy, $\delta x, y \sim E_{\gamma}^{-1 / 2}$, so high energy photons contribute most to the resolution.

The collimators must have hard edges for optical diffraction, to prevent showers from leaking thru. Since photons are attenuated like $e^{-z / L_{R}}$, thick, high $\mathrm{Z}$ mirrors ( $W$ or $\mathrm{Ta}$ ), with short radiation length, $L_{R}$, should permit very little transtnission, $e^{-12} \sim 10^{-5}$. In addition, the body of the primary collimitor and slit must be shaded (shielded) to reduce the absorbed heat from showers, and this would significantly reduce the incident and transmitted photon flux.

An option for the bremsstrahlung detector is shown in Fig 6, with pair converter followed by Cherenkov radiator. Sweeping magnets may be required to reduce shower background. The total number of pair produced leptons $n_{e, p a i r}=n_{e, p r i m a r y}\left(l / L_{R}\right) \eta\left(\phi / \sigma_{\pi^{\prime}, \gamma}\right)$, where $n_{c}$ is the number of electrons, $l / L_{R}$ is the thickness of the bremsstrahlung radiator in radiation lengths $, L_{R}, \eta$ is the iumber of electrors detected for one equivalent 
full energy photon on the detector, which must be calculated by monte carlo and is roughly 1 - 10 depending on detector, $\phi / \sigma_{x^{\prime} \gamma}$ is the acceptance of the detector slit divided by the divergence of the photon beam. In fact, the monte carlos calculate the flux of pair produced leptons of desired angle and energy for a given number of incident beam electrons, integrating over bremsstrahlung angle.
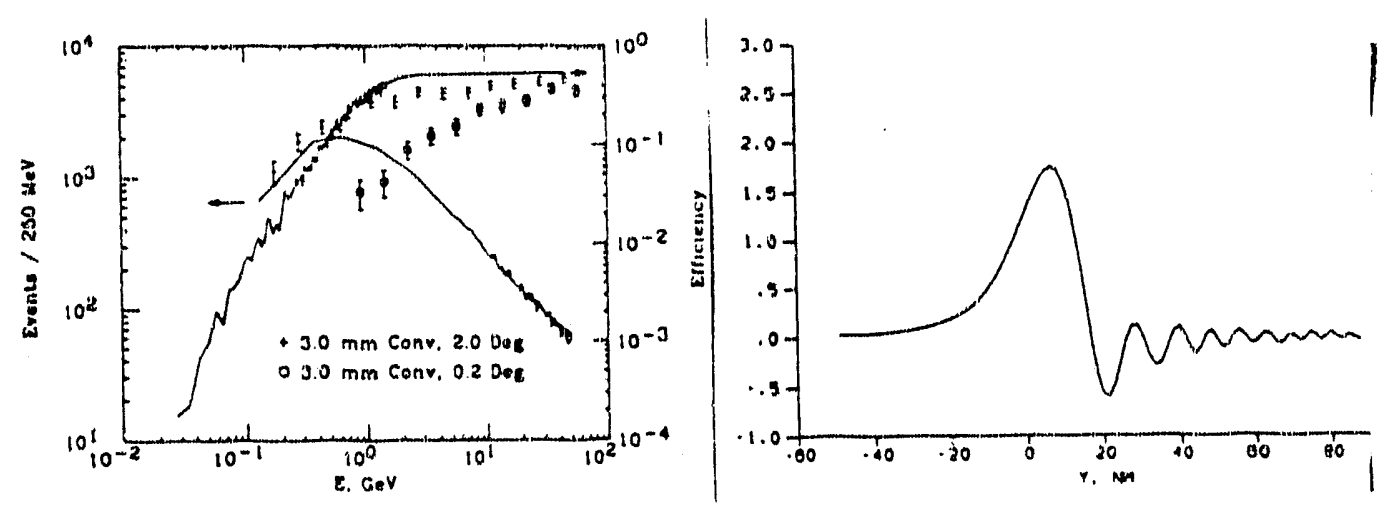

Figures 4 and 5. Monte Carlo simulations of the detection efficiency and photon spectrum for different pair acceptance angles, along with the resolution function for $\left(E_{e}=50 \mathrm{GeV}, a=2, b=20 \mathrm{~m}, \theta_{\text {pair }}=0.2^{\circ}\right)$.

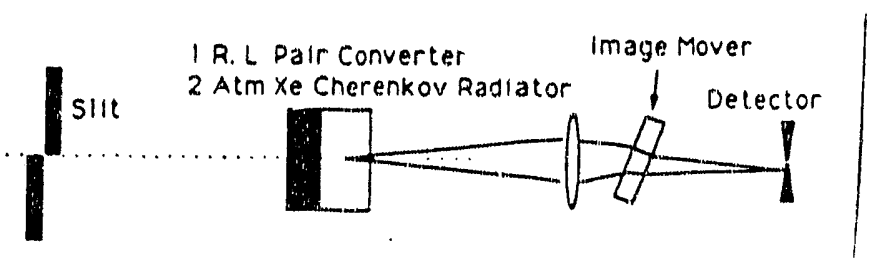

Figure 6. One option for a bremsstrahlung detector. Sweeping magnets and distance may be useful in separating the low emittance signal from showers.

The number of detectable photons would then be $n_{\gamma} \sim\left(150_{[1 / \mathrm{cm}]} n_{e, p a i r} L_{(\mathrm{cm}]} \sin ^{2} \theta_{C},[8]\right.$ which would yield $\sim 2000$ photons in one Fresnel half width. It is assumed that Xe gas at $1 \mathrm{~atm}$ can be used as the Cerenkov radiator. With a refractive index $n=1.00071$, the opening angle of radiation is $2.1^{\circ}$, and the minimum detectable electron energy is $\sim 12$ $\mathrm{MeV}$. This paper assumes that the pair converter is $U 3.6 \mathrm{~mm}$ thick and the $C$ radiator is $2 \mathrm{~cm}$ thick, and the combined width due to pair production / shower dimensic ns and Cerenkov optics is $\sim 100 \mu$.

It is somewhat difficult to determine the longitudinal position of the wasis since this system only sees roughly horizontal slices of the phase space at the focus. The measurement can be made by moving the primary collimator by a large distance $2 a \sigma_{y^{\prime}} \sim 4 \mathrm{~cm}$, measuring the position of the mean of of the penumbra at two points and extrapolating 
back to the focus. the error on the focal position is then $\Delta z=\sqrt{2} \delta y / \sigma_{y^{\prime}} \sim 140 \mu \sim \beta_{y}^{*}$, where $\delta y=100 \mathrm{~nm}$ is the measurement error.

Multiple scattering broadens the beam of electrons as they traverse the bremsstrahlung radiator. This effect can be evaluated by Monte Carlo, assurning a scittering angle of $\theta_{m s}=13.6[M e V] \sqrt{d x / L_{R}} / p_{e,[M e V]}$, where $d x / L_{R}$ is a path length element in radiation lengths. In general the multiple scattering correction is small as long as the change in beam size due to multiple scattering, $s$, is small compared to the unpertusbed size.

Self fields will focus the beam and cause it to radiate. At SLAC, magnetic fields $B_{\phi} \sim$ $\mu_{0} c Q / 4 \sqrt{2} \pi \sigma_{x} \sigma_{z} \sim 50 T$ will produce deflections, $\theta_{f, \max } \sim B_{\phi} l / B \rho=0.0002$. The field $B_{\phi}$ will cause synchrotron radiation, however the energy seen by the detector can be estimated from $\left(\theta_{f, \max }\right) \times($ loss/turn $)$ for electrons in this field, $d E_{[\mathrm{GeV} / \mathrm{turn}]}=8.8$. $10^{-5} \times E_{e,[G e V]}^{4} / P_{[m]}$, which is about $0.004 \mathrm{~J}$ for $3 \cdot 10^{10}$ electrons. With a critical energy of $E_{c_{c},[\mathrm{GeV}]}=6.6 \cdot 10^{-7} \times E_{e_{1}[\mathrm{GeV}]}^{2} B_{\phi_{1},[T]} \sim 91 \mathrm{MeV}$, most photons are $100 \mathrm{MeV}$ or less. The total energy lost into this radiation will be much smaller than into bremsstrahlung. Total energy for bremsstrahlung is $\sim 24 \mathrm{~J} /$ pulse, and when the detection efficiency is considered the total energy in synchrotron photons is $\sim 0.1 \%$ of the bremsstrahlung energy.

The intense electron beam will ionize the bremsstrahlung radiator and these heavy ions will then be pushed toward the median plane of the electron beam, which will significantly alter the local radiation length. Ionization has been calculated assuming $\sigma_{+,(M b]} \sim 0.12 Z+0.2$ or $\mathrm{Mb}$ for $\mathrm{Pb},[4][9]$ with $\sigma_{++}=\sigma_{+} / 4, \sigma_{+++}=\sigma_{++} / 4 .[10]$ Figure 7 shows the result that the $\mathrm{Pb}$ is fully ionized, and significant double and triple ionization also occurs. Ion motion has been calculated and is significant. The ions are accelerated to energies of about a keV and then travel toward the median plane of the beam, where they interpenetrate to create what seems to be a very high density for a short time. One effect of this ion motion will be a hole in the radiator, which must be moved for subsequent pulses. For electron pulse trains, a liquid metal jet, of the sort developed by $F$. Villa [11] could be used as a bremsstrahlung radiator.

Figure 7. The ionization state

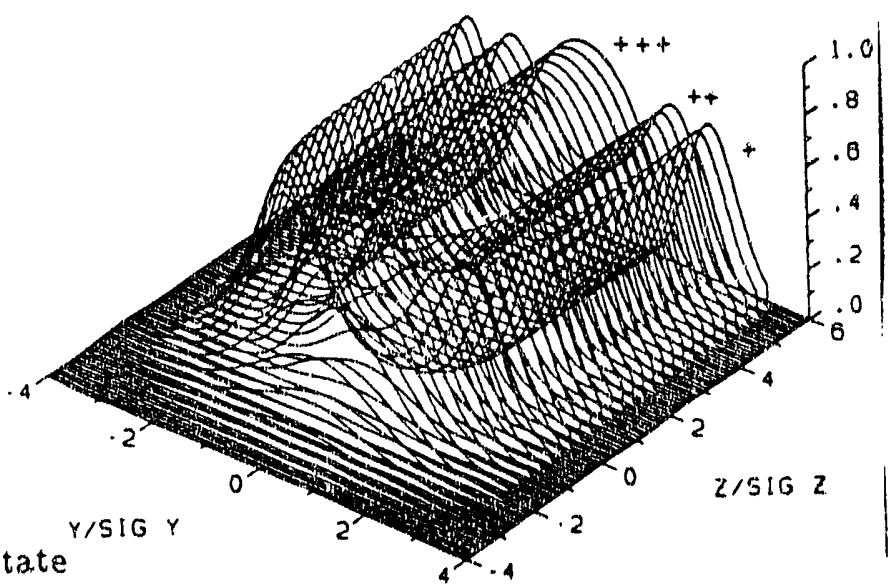
of atoms in the heavy bremsstrahlung target.

COMPONENTS 
It is assumed that high $\mathrm{Z}$ materials will be used for the foil, and that these will be locally destroyed by the $1 \mathrm{~mJ}$ of ionization energy deposited on every pulse. If a large foil area was inserted and loosely mounted from some point upstream or downstream of the focus, mechanical rastering the foil should be sufficient to provide a new part of the foil for every bunch, while maintaining the position along the beam line. The foil thickness should be less than $\beta^{*}$.

Precision collimators can be made from commercially available metal mirrors, which are described in catalogues with a flatness of $\sigma<\lambda / 20 \sim 24 \mathrm{~nm}$, and surface roughness of better than $1 \mathrm{~nm}$. Synchrotron light sources require microroughness in the range of 0.2 $\mathrm{nm}$ and slope errors in the range of 0.1 arc second. Metal mirrors with dimensions of $\sim 5 \cdot 10 \mathrm{~cm}$, optically polished to a few nm surface roughness, would cost about $\$ 1000$ - 2000, and take about 10 weeks to fabricate.[12][13]. With glass mirrors, requiring $\sigma<\lambda / 200$ adds about $50 \%$ to the cost relative to $\lambda / 40$.

Since the primary collimators must be thermally and mechanically stable and must be moved with high precision, it is difficult to simultaneously design them to absorb significant beam power. The guard collimators on the other hand should be able to absorb this power, while maintaining alignment only to the level of $\sim 0.1 \mu$. Rough alignment can be done with standard techniques using transit and levels. Alignment of the collimator surfaces directly parallel to the beam can be done with optical lenses and prisms, which have angular tolerances of \pm 30 arc seconds, or $0.14 \mathrm{mrad}$. More precise alignment of the collimators would probably require the beam on target. Slits can be produced using two single collimators, oftset to eliminate collisions.

Rough positioning of collimators can be done with a number of commercially available systems, such the the Nanomover sold by Melles Griot, which can set $20 \mathrm{~kg}$ loads with $\pm 100 \mathrm{~nm}$ resolution over $25 \mathrm{~mm}$.[12] The primary collimator and final slit would have to be more carefully positioned, possibly to tolerances of $\pm 1 \mathrm{~nm}$. It is assumed that the collimators will each be controlled with three actuators, and the precision adjustment of these would be done in real time. Mirrors can be mounted to the structure in a number of ways, a compliant mount, such as pitch, might be desirable.

Since ground vibrations occur at the level of about $\sigma_{v} \sim 0.035 \mu, \sigma_{h} \sim 0.1 \mu$, the beam defining collimators must at least be stabilized against the vertical motion.[15] Although other options are available, The Streckeisen STS-2, while somewhat expensive, is suffciently sensitive, is linear in amplitude. The velocity and phase response are straightforward for periods from 0.03 to 300 seconds, which spans the low frequency range where ground vibration is largest. An open loop correction system using this system should be able to correct the collimator and/or slit positions to better than $1 \mathrm{~nm}$ over the range 0.1 to $10 \mathrm{~Hz}$.

Additional shielding is required for a number of problems: backgrounds in the detectors, heating on the collimators and support frames, activation of the seismometers, which must be installed near the collimators, and minimizing radiation levels outside the shielding. In principle these are problems which can be solved using standard procedures. Considerable spray is produced by the electron beam in the target and this will be a significant source of shower secondaries. 
Since the FFTB is being shielded for $\sim 2.5 \mathrm{~kW}$ of beam power and the bremsstrahlung radiator is anticipated to be 0.1 radiation lengths thick, it is likely that the shielding and cooling of the collimator assemblies must be able to deal with about $250 \mathrm{~W}$ of bearn power. If low expansion materials are used in structural applications, thermal expansion of components could significantly affect the alignment, critical components must be designed either to absorb power or to avoid the beam spray. It is anticipated that local shielding can protect the majority of the apparatus from the scattered beam however some moveable guard collimators will likely absorb significant heat which will require a total cooling capacity of $\sim 0.25 \mathrm{~kW}$.. In a simple test, a piezocrystal was used

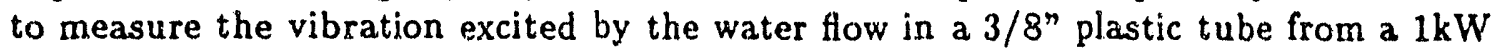
cooling unit. Although large amplitude vibrations were seen from a large number of sources, (movement, pumps etc), the cooling water flowing thru a water fitting caused less than $1 \mathrm{~nm}$ of motion.

\section{EXPERIMENTS}

A preliminary experiment is being done using the low energy beams of the APS injector at Argonne to help optimize the detector and mechanical systems. This test will look at the beam on the positron production target from downstream of the positron linac. This test should permit optimization of shielding, detectors, nechanical systenls and control algorithms.

The first real test should be on the SLAC/FFTB, where beam sizes of $60 \mathrm{~nm}$ will be produced. This size is comparable with the spot sizes required by the TESLA design, but somewhat larger than spotsizes required by some collider designs[16] [1]. Other possible applications of the proposed system would be plasma focussing experiments, measurements of beams from nonlinear QED experiments, measurements of drifts and jitter, and multibunch stability.

\section{COLLIDER}

If this technique is used for single beams in the final detector of a linear collider it will be necessary to insert a thin radiator of some kind near the center of the large high energy physics detector, Fig 8.[17] Ideally this radiator can be quite small and light, and, since the $z$ position needs to be determined only to some fraction of $\beta^{*}$, the required positioning tolerances are not challenging. While the design of the target holder, collimators and detector would pose significant problems, the most significant interaction with the detector design may be the spray from the bremsstrahlung target.

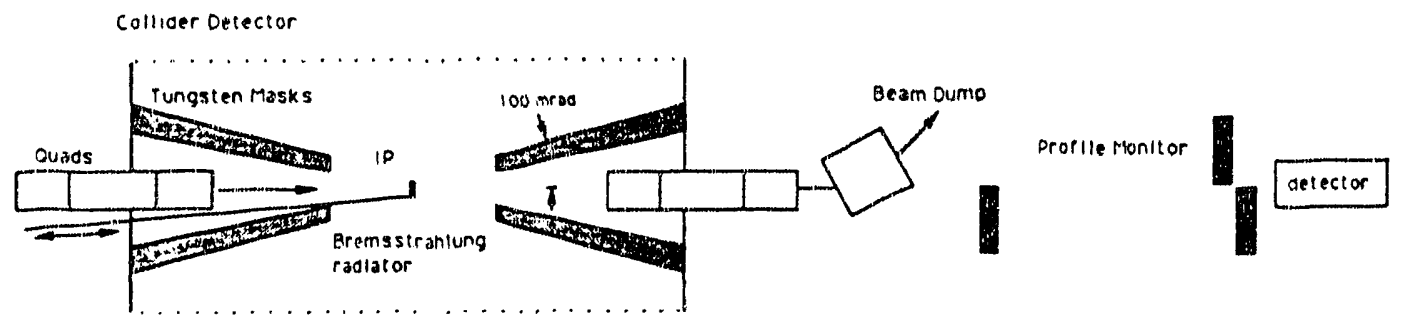

$\square$ Figure 8. Schematic of operation with a collider detector. 
While a high $\mathrm{Z}$ bremsstrahlung target at the IP would be a copious source of shower secondaries and radiation damage, the magnitude and composition of these showers should be somewhat similar to showers produced by beamstrahlung from $e^{+} / e^{-}$collisions. In normal operation, beamstrahlung will convert a significant fraction, $\delta$, of the total energy of beam bunch into secondaries. Various designs give $\delta=0.1-0.3$ for high frequency, $(12 \mathrm{GHz})$, options,[1], and $\delta=0.01-0.1$ for low frequency options, $(1.5$ $\mathrm{GHz}$ ), such as TESLA[16]. By comparison a bremsstrahlung target would approximate an energy loss of $\delta<\beta^{\star} / L_{R} \sim 0.03-0.1$. Designs for collider detectors include tungsten masks to prevent low energy beamstrahlung secondaries emitted at angles of less than $100 \mathrm{mrad}$ from entering the detector and these masks should also be useful for bremsstrahlung.

In principle, beamstrahlung from collisions could be used to provide direct images of the colliding bunches using this technique. It may be difficult, however, to get high statistics and high resolution if the collider is run in a mode where the average beamstrahlung photon energy is a small fraction, $\Upsilon$, of the incident electron energy. Values of $\Upsilon$ vary widely, $\sim 0.01-0.6$, from design to design, and should be much lower during tune up, but the method should be useful.

\section{CONCLUSIONS}

A single bunch, beam profile monitor using bremsstrahlung should be capable of very high resolution when used with the high energy electron beams associated with linear colliders. The technique uses comparatively simple optics and inexpensive components and seems compatible with operation in linear collider detectors.

\section{REFERENCES}

[1] R. B. Palmer, Annu. Rev. Nucl. Part. Sci. 40, 529, ('90)

[2] J. Norem, Proceedings of the 1988 Linear Accelerator Conf., Newport News, Virginia, (1988), p430

[3] K. Oide, Proc. 1989 Particle Accelerator Conf, Chicago, 1989, p1319

[4] J. Buon, F Couchot, J. Jeanjean, F. Le Diberder, V Lepeltier, H. Nguyen Ngoc, J. Perez-y-Jorba, M. Puzo, and P. Chen, Nucl. Instrum. and Methods., A306, (1991), 93

[5] T. Shintake, Nucl. Instrum. and Methods, A.311, (1992), 453

[6] Jenkins and White, Fundamentals of Optics, Fresnel Diffraction. McGraw-Hill (1957)

[7] H. A. Bethe, in Experimental Nuclear Physics Vol 1, E. Segre, ed., John Wiley and Sons Inc. New York, (1953), p166

[8] J. Litt and R. Muenier, Annu. Rev. Nucl. Part. Sci., 23, 1, ('73) 
[9] P. Chen, Contribution to NLT Workshop SLAC, (1988)

[10] B. Rossi, High Energy Particles, Prentice-Hall Inc, Englewood Cliffs N. J., (1952)

[11] F. Villa, (SLAC, private communication, 1991)

[12] Melles Griot, Irvine Calif. 92714

[13] R Lowrey, Rockwell Power Systems, Albuquerque NM (private communication)

[14] J. B. Rosenzweig, P. Schoessow, B. Cole, C. Ho, W. Gai, R. Konecny, S. Mtingwa, J. Norem, M. Rosing, and J. Simpson, Phys. Fluids B 2, 1376, (1990)

[15] R. E. Ruland, G. E. Fischer, Proceedings of the 2nd International Workshop on Accelerator Alignment, Hamburg, Germany, Sept 10-12, 1990. and Stanford Linear Accelerator Laboratory publication SLAC-PUB-5326 (1990)

[16] Proc. of the 1st TESLA Workshop, Cornell U, Ithaca, NY, CLNS 90-1029 (1990), ed $\mathrm{H}$ Padamsee.

[17] Schroeder, D. V., Stanford Linear Accelerator Center Report SLAC-371, SLAC, Stanford, CA, (1990)

\title{
DISCLAIMER
}

\begin{abstract}
This report was prepared as an account of work sponsored by an agency of the United States Government. Neither the United States Government nor any agency thereof, nor any of their employees, makes any warranty, express or implied, or assumes any legal liability or responsibility for the accuracy, completeness, or usefulness of any information, apparatus, product, or process disclosed, or represents that its use would not infringe privately owned rights. Reference herein to any specific commercial product, process, or service by trade name, trademark, manufacturer, or otherwise does not necessarily constitute or imply its endorsement, recommendation, or favoring by the United States Government or any agency thereof. The views and opinions of authors expressed herein do not necessarily state or reflect those of the United States Government or any agency thereof.
\end{abstract}




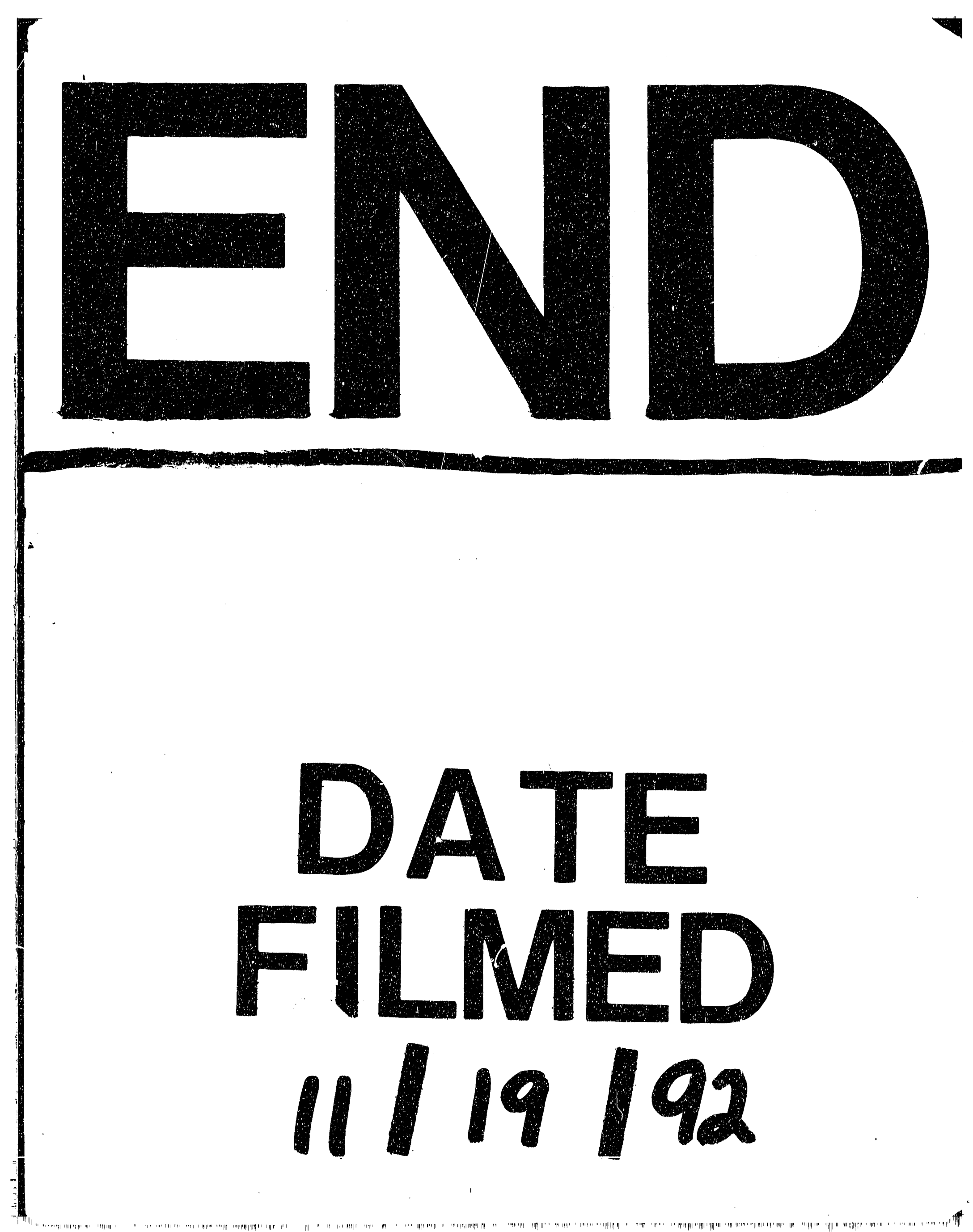


